\title{
Effects of bone marrow-derived mesenchymal stem cell transplantation on chronic obstructive pulmonary disease/obstructive sleep apnea overlap syndrome in rats
}

\author{
MIN CHEN $^{1 *}$, ZHAOMING HUANG $^{1 *}$, HONG BI $^{1}$, XINGHUA PAN $^{2}$, JIAN HE $^{1}$, LEWEI HE ${ }^{1}, \mathrm{XU} \mathrm{HE}^{1}$, \\ JUNYI DU $^{1}$, KAIHUA ZHOU ${ }^{1}$, LIYAN WANG ${ }^{1}$, QING WANG $^{1}$, XIANG GUO ${ }^{1}$ and ZHIXIAN JIN ${ }^{1}$ \\ ${ }^{1}$ Department of Pneumology, The First People's Hospital-Calmette Hospital of Kunming, Kunming, Yunnan 650224; \\ ${ }^{2}$ Medical Innovation Research Center, 920 Hospital of PLA Joint Logistics Support Force, \\ Kunming, Yunnan 650032, P.R. China
}

Received April 11,2019; Accepted September 4, 2019

DOI: $10.3892 / \mathrm{mmr} .2019 .10714$

\begin{abstract}
Bone marrow-derived mesenchymal stem cells (BMSCs) possess potential therapeutic properties for treating patients with chronic obstructive pulmonary disease (COPD), which is characterized by emphysema and obstructive sleep apnea (OSA). However, their effects on overlap syndrome (OS) remain unclear. We investigated the potential therapeutic effects and possible mechanisms of BMSC transplantation in OS rats. To generate the OS model in rats, the animals underwent daily exposure to cigarette smoke and intermittent hypoxia. BMSCs were intravenously injected into rats. At 4 weeks post-transplantation, the severity of emphysema was assessed by lung hematoxylin and eosin (H\&E) staining. The levels of oxidative stress and the malondialdehyde (MDA) and superoxide dismutase (SOD) contents in serum and lung were detected. The apoptosis of alveolar septal cells was also detected by TUNEL assay. Finally, we determined the expression of CD31 and VWF in lung tissues by an immunohistochemical (IHC) assay. It was found that BMSCs were able to migrate to the injured lung and aorta tissues. In lung tissues, transplanted BMSCs, some of which had differentiated into endotheliocytes, were found in the alveolar walls. The mean linear intercept (MLI) and pathological scores were higher and the mean alveolar number (MAN) was lower in the OS group than these parameters in the control group. These values were significantly reduced in the OS+BMSC group
\end{abstract}

Correspondence to: Professor Zhixian Jin, Department of Pneumology, The First People's Hospital-Calmette Hospital of Kunming, 1228 Beijing Road, Kunming, Yunnan 650224, P.R. China E-mail: jzx19706699@hotmail.com

${ }^{*}$ Contributed equally

Key words: chronic obstructive pulmonary disease, obstructive sleep apnea, overlap syndrome, emphysema, mesenchymal stem cells, transplantation, endotheliocytes, oxidative stress, apoptosis compared to those in the OS group. The MDA content was decreased and SOD activity was increased in the OS+BMSC group compared to those in the OS group. The apoptotic index of alveolar wall cells in the OS group was higher than that in the OS+BMSC group. The expression levels of CD31 and VWF in alveolar wall cells in the OS group were lower than those in the OS+BMSC group. These results indicate that BMSCs may inhibit the progression of emphysema in the OS model by differentiating into endotheliocytes and suppressing the apoptosis of endotheliocytes and oxidative stress. There is a possibility that the release of growth factors and structural support may be a determinant for the regenerative effects observed following treatment with BMSCs.

\section{Introduction}

Chronic obstructive pulmonary disease (COPD) and obstructive sleep apnea (OSA) are the most prevalent chronic respiratory disorders in the world (1). COPD is progressive and most commonly arises from cigarette smoking and exposure to pollutants. It is characterized by persistent respiratory symptoms and nonreversible air-flow limitation (2). Obstructive sleep apnea (OSA) is defined by repeated episodes of upper airway occlusion, which results in brief periods of breathing cessation (apnea) or a marked reduction in flow (hypopnea) during sleep (3). David Flenley (4) was the first to coin the term 'overlap syndrome' (OS) meaning the coexistence of COPD with OSA. Overlap syndrome has an increased mortality compared with either COPD or OSA alone. Marin et al (5) found a decreased survival rate among patients with OS compared with either COPD or OSA alone. Data are emerging that show worse deleterious cardiac changes in individuals with OS compared to those with COPD alone $(6,7)$. Although much research still needs to be conducted, more and more researchers are vigilant about OS.

Bone marrow-derived mesenchymal stem cells (BMSCs) are pluripotent stem cells that can differentiate into osteoblasts, chondrocytes or muscle cells and even into nerve cells or hepatocytes (8). BMSCs have a good paracrine function, enabling them to secrete a variety of substances, such as neurotrophic 
factors, cytokines and chemokines to regulate the local microenvironment of damage, reduce inflammatory response, and promote the repair of damaged tissues. MSCs have potential therapeutic roles in chronic lung diseases (9). MSCs have the ability to regulate the immune response to tissue injury, and MSCs also promote repair in vivo and have been suggested as an attractive therapeutic candidate for various types of lung diseases, including chronic obstructive pulmonary disease (COPD), among others (10). Studies involving animal models have already demonstrated the effect of MSCs on tissue regeneration after elastase-induced emphysema, asbestos or endotoxin-induced lung injury $(11,12)$. OSA can induce oxidative stress, inflammation and endothelial dysfunction, along with certain clinical consequences such as cardiovascular diseases and neurocognitive alterations (13-15). MSCs play an important role in the physiological response to OSA (16). MSCs also have potential therapeutic effects on COPD $(17,18)$.

Emphysema is an important pathological characteristic of COPD, characterized by a loss of alveolar walls and permanently enlarged cavities of the terminal bronchioles (19). Oxidative stress plays an important role in the pathogenesis of COPD (20-23). The increased apoptosis in alveolar cells of the COPD model and MSCs can inhibit the apoptosis of alveolar cells $(24,25)$. Although it has been reported that MSCs play a protective and reparative role in COPD and OSA, the data currently available on the potential role of BMSCs in overlap syndrome (OS) are still scarce. Moreover, the possible mechanisms of MSCs in OS have not been reported.

In this study, rats were used to generate the OS model by daily exposure to cigarette smoke and intermittent hypoxia. To investigate the effects and possible mechanisms of BMSCs on the OS model, BMSCs were intravenously injected into OS model rats. We found that BMSCs were able to alleviate lung injury in OS rats. Moreover, BMSCs inhibited oxidative stress and the apoptosis of endotheliocytes. The experiment validated the antioxidative stress and antiapoptotic effect of BMSCs in an OS model.

\section{Materials and methods}

Isolation and culture of BMSCs. The extraction and characterization methods of BMSCs in rats were performed as described previously (26). A total of 3 one-week-old Sprague-Dawley (SD) rats (Vital River) were sacrificed by cervical vertebra luxation. The femur and tibia of rats were removed under sterile conditions. Bone marrow cells of rats were collected from the femurs and tibias with Dulbecco's modified Eagle's medium (DMEM; HyClone; GE Healthcare). The DMEM containing bone marrow cells was added to a Percoll gradient (Pharmacia Biotech; GE Healthcare) and centrifuged at $270 \mathrm{x}$ g for $30 \mathrm{~min}$. The mononuclear cells were harvested and added to DMEM containing $10 \%$ fetal bovine serum (FBS; Gibco/Thermo Fisher Scientific, Inc.). The cells were cultured at $37^{\circ} \mathrm{C}$ in a $5 \% \mathrm{CO}_{2}$ incubator. After $24 \mathrm{~h}$, nonadherent cells were removed, and the medium was replaced every 3-4 days. MSCs from the third passage were used in the subsequent experiments. BMSCs were cultured and passaged with DMEM/F12 medium (HyClone; GE Healthcare) containing $10 \% \mathrm{FBS}, 100 \mathrm{U} / \mathrm{ml}$ penicillin and $0.1 \mathrm{mg} / \mathrm{ml}$ streptomycin.
Identification of molecular markers on the surface of the $B M S C s$. Third-generation cells were digested with trypsin (HyClone/GE Healthcare), washed with PBS 1-2 times, and then centrifuged to collect the cells. A total of $1 \times 10^{6}$ cells were taken, and $350 \mu \mathrm{l}$ of $1 \mathrm{X}$ PBS was added. After mixing, flow tubes were added at $50 \mu \mathrm{l} /$ tube. Next, $2 \mu \mathrm{l}$ of a fluorescence-labeled monoclonal antibody CD19-Fluor 488 (cat. \#NBP2-24965AF488, Novus Biologicals, USA), CD45-FITC (cat. \#85-11-0461-80, eBioscience), CD29-FITC (cat. \#85-11-0291-82, eBioscience), CD90-FITC (cat. \#85-11-0900-85, eBioscience) or isotype control FITC (cat. \#85-11-4031-81, eBioscience) was added. Next, $48 \mathrm{ml}$ PBS was added to each tube and incubated at room temperature in the dark for $30 \mathrm{~min}$. Cell surface markers were detected by flow cytometry (Sysmex Partec GmbH).

GFP-labeled BMSCs. Before infection, the eGFP-lentivirus (GM100202-2, Shanghai Genomeditech Co., Ltd.) was diluted in complete medium according to the manufacturer's instructions. The old medium was removed, and the virus described above and polybrene were added to the cells for infection and bleeding. After $48 \mathrm{~h}$ of infection, the medium containing virus was replaced with fresh medium for further culture. After $48 \mathrm{~h}$, cells were injected intravenously into rats. Four weeks after the last injection, the BMSC location was observed under a confocal fluorescence microscope (Leica Microsystems $\mathrm{GmbH})$.

Establishment of the OS model by cigarette smoke and intermittent hypoxia. The OS model was established by the cigarette smoke (27) and intermittent hypoxia exposure method. A total of 24 SD rats (female, 150-200 g, 6-week-old) were raised under controlled temperature $\left(19-23^{\circ} \mathrm{C}\right)$ and humidity (40-60\%), and maintained on a 12-h light/dark cycle lights. Food and water were provided ad libitum. All animal experiments were approved by the Institutional Animal Care and Use Committee of Kunming Medical University. Rats in the OS group were exposed to cigarette smoke (15 cigarettes once, 2 times daily) and intermittent hypoxia (30 times per h, 8 h per day) exposure for 8 weeks.

Grouping and BMSC transplantation. Female rats were randomly divided into three groups $(n=8)$ : Control, OS and OS+BMSCs. Rats in the control group were treated with false smoke and air exposure. From $24 \mathrm{~h}$ after cigarette smoke and intermittent hypoxia exposure, the rats in the OS group were injected intravenously with PBS $(50 \mu \mathrm{l})$ and those in the OS+BMSC group were injected intravenously with $50 \mu \mathrm{l}$ of MSCs (total number of cells: $2 \times 10^{6} / \mathrm{rat}$ ) via the tail vein (1 time/week on the 7 th day of each week, total 4 times). Three of the OS model rats were injected intravenously with the GFP-labeled BMSCs to investigate the location and differentiation of the transplanted BMSCs, and five of the OS model rats were injected intravenously with unlabeled BMSCs for examination with the TUNEL assay. All rats were used to perform $\mathrm{H} \& \mathrm{E}$ staining and immunocytochemistry assays.

Lung morphologic analysis, quantification of emphysema and lung injury score. On week 4 of the last BMSCs injection, all rats were euthanized with an overdose of anesthetic (3\% sodium pentobarbital $180 \mathrm{mg} / \mathrm{kg}$, i.p.). Lung tissues were fixed in $4 \%$ 
formalin, embedded in paraffin, cut into $4-\mu$ m-thick sections, and stained with hematoxylin and eosin (H\&E). Histological assessment of the sections was determined using the mean alveolar number (MAN) and the mean linear intercept (MLI) method as previously described $(28,29)$ by Image-Pro Plus 6.0 software (Media Cybernetics, Inc.). Three visual fields were observed in each slice, in which no trachea or large blood vessels were observed. The ' + ' symbol was located in the middle of each field of each section; the number of alveolar intervals (NS) that passed the ' + ' symbol was determined, the total length $(\mathrm{L})$ of the ' + ' symbol was measured $(\mathrm{mm})$, the area of the field was determined (S), and the number of alveola $(\mathrm{Na})$ in each field was counted (MLI=L/NS and $\mathrm{MAN}=\mathrm{Na} / \mathrm{S}$ ). The pathological scores were detected as a reference (30). Medium size $(300-1100 \mu \mathrm{m})$ membranous bronchi were selected to observe the following indicators: i) epithelium abscission, erosion and ulcer formation; ii) hyperplasia and hypertrophy of epithelial goblet cells; iii) mucosal epithelial ciliary lodging; iv) inflammatory cell infiltration and exudation of the airway wall; v) the formation of lymphatic nodules in the vessel wall; vi) stenosis of the small airway lumen; vii) a proliferation disorder of respiratory smooth muscle; viii) connective tissue hyperplasia of the airway wall; ix) squamous metaplasia of mucosal cells; $x$ ) wall congestion and edema; and xi) pigmentation on the wall. Each item was scored 0, 1 for mild, 2 for moderate, or 3 for severe. Three membranous bronchioles were randomly selected from the pathological sections of each rat.

Determination of SOD and MDA levels. The contents of SOD (xanthine oxidase assay method) and MDA (thiobarbituric acid assay method) were measured using respective kits purchased from Nanjing Jiancheng Bio-Engineering Institute Co., Ltd. (according to the manufacturer's instructions).

TUNEL assay. The apoptosis of alveolar septal cells was also detected using a TUNEL assay kit (cat. no. 11684817910 , Roche Diagnostics). Tissue samples were fixed in $4 \%$ formaldehyde for $24 \mathrm{~h}$ and embedded in paraffin. Next, $4-\mu \mathrm{m}$-thick paraffin sections were adhered to slides. Sections were deparaffinized by heating the slides for $30 \mathrm{~min}$ at $60^{\circ} \mathrm{C}$ (or $10 \mathrm{~min}$ at $70^{\circ} \mathrm{C}$ ), followed by two 5 -min incubations in a xylene bath at room temperature. The tissue samples were rehydrated by transferring the slides through a graded ethanol series: $2 \times 3$ min $90 \%$ ethanol, $1 \times 3$ min $80 \%$ ethanol, $1 \times 3$ min $70 \%$ ethanol, and $1 \times 3$ min double-distilled water $\left(\mathrm{ddH}_{2} \mathrm{O}\right)$. Excess water was carefully blotted away, and $20 \mathrm{mg} / \mathrm{ml}$ proteinase $\mathrm{K}$ solution was pipetted to cover the sections. The mixture was then incubated for $15 \mathrm{~min}$ at room temperature. Following proteinase $\mathrm{K}$ treatment, the slides were washed $3 \times 5 \mathrm{~min}$ with $\mathrm{ddH}_{2} \mathrm{O}$. Then, the sections were covered with $50 \mu \mathrm{l}$ of TUNEL reaction buffer for $60 \mathrm{~min}$ at $37^{\circ} \mathrm{C}$. The slides were washed with PBS and air dried. The tissue sections were examined by confocal microscopy (magnification, x400).

Immunofluorescence. Fixed tissues were dehydrated with various concentrations of xylene and ethanol $(50 \%$ ethanol for $4 \mathrm{~h} ; 75 \%$ ethanol for $4 \mathrm{~h} ; 85 \%$ ethanol for $3 \mathrm{~h}$; $95 \%$ ethanol for $2 \mathrm{~h}$; $100 \%$ ethanol for $1 \mathrm{~h} ; 100 \%$ ethanol for $1 \mathrm{~h} ; 1: 1$ ethanol-xylene for $1 \mathrm{~h}$; xylene for $1 \mathrm{~h}$; and xylene for $30 \mathrm{~min}$ at room temperature) and embedded in paraffin. Sections
(4- $\mu \mathrm{m}$ thickness) were cut from a paraffin block. Sections were dewaxed with various concentrations of xylene and ethanol (xylene for $10 \mathrm{~min}$; xylene for $5 \mathrm{~min}$; $100 \%$ ethanol for $5 \mathrm{~min}$; $95 \%$ ethanol for $2 \mathrm{~min}$; $80 \%$ ethanol for $2 \mathrm{~min}$; and $70 \%$ ethanol for $2 \mathrm{~min}$ ). Antigen retrieval was performed on the sections with $0.01 \mathrm{M}$ citric acid buffer $(\mathrm{pH} 6.0)$ at $100^{\circ} \mathrm{C}$ and $80 \mathrm{kPa}$ pressure. Sections were blocked by incubation with $5 \% \mathrm{v} / \mathrm{v}$ normal goat serum (cat. no. SL038, Beijing Solarbio Science \& Technology Co., Ltd.) in PBS for $15 \mathrm{~min}$ at room temperature. Sections were incubated with an anti-CD34 antibody (dilution 1:100, cat. no. A10796; ABclonal Biotech Co., Ltd.) overnight at $4^{\circ} \mathrm{C}$ and with a CoraLite594-conjugated secondary antibody (dilution 1:100; cat. no. SA00013-4; ProteinTech Group, Inc.) for $2 \mathrm{~h}$ at room temperature. Nuclei were stained with $10 \mu \mathrm{g} / \mathrm{ml}$ DAPI in the dark for $5 \mathrm{~min}$ at room temperature. Sections were observed using a fluorescence microscope (magnification, x200). Three representative fields of stained cells were analyzed using Image-Pro Plus software (version 6.0; Media Cybernetics, Inc.) to obtain the mean optical density (OD), which represents the staining strength per positive pixel.

Immunocytochemistry. Sections were incubated with an anti-CD31 antibody (dilution 1:100, A3181; ABclonal) or an anti-VWF antibody (dilution 1:100, A1335; ABclonal) overnight at $4^{\circ} \mathrm{C}$ followed by incubation with goat anti-rabbit IgG $(\mathrm{H}+\mathrm{L})$ horseradish peroxidase (HRP)-conjugated secondary antibody (dilution 1:200, AS014, ABclonal) for $2 \mathrm{~h}$ at room temperature. The reactions were visualized using a 3,3'-diaminobenzidine visualization kit (Fuzhou Maixin Biotech Co., Ltd.). Sections were counterstained with hematoxylin to visualize nuclei, stained with hematoxylin for 5-10 $\mathrm{min}$ at room temperature, and dehydrated. Sections were examined under a light microscope at a magnification of x200. Brown staining indicated immunoreactive positive cells, and blue staining indicated the nuclei.

Statistical analysis. All values are expressed as the mean \pm SD. Comparisons were assessed with one-way analysis of variance (ANOVA) (Tukey's post hoc test) by using GraphPad Prism software version 5.0a (GraphPad, USA). All experiments were conducted at least three times. $\mathrm{P}<0.05$ was considered statistically significant.

\section{Results}

Identification of BMSCs. As shown in Fig. 1A, BMSCs exhibited shapes similar to stars and spindles, with round nuclei in the center, cytoplasmic hypertrophy and a clear membrane. Flow cytometry detection showed that the third-generation BMSCs expressed high levels of CD29 (99.9\%) and CD90 (99.9\%), and low levels of CD19 (1.0\%) and CD45 (3.1\%) (Fig. 1B). These results indicate that rat BMSCs were successfully collected.

Location and differentiation of BMSCs transplanted in the lung tissue. The OS model was established by exposure to cigarette smoke and intermittent hypoxia. The model profile for each day is shown in Fig. 2A. At the beginning of the smoke exposure, the rats were agitated, irritable and pulsating, followed by less movement, eye closing, wheezing, salivating 
A

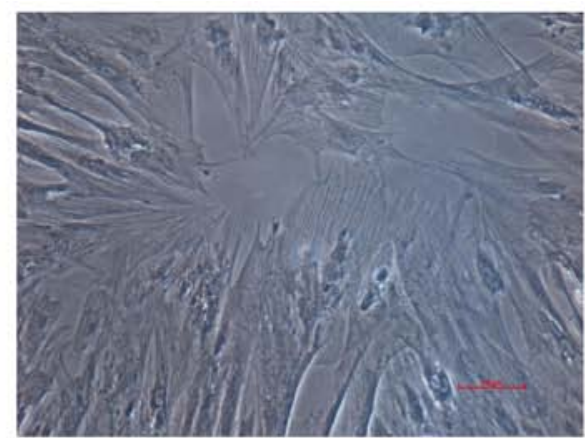

B

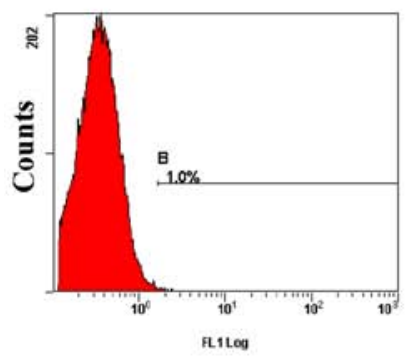

CD19
(FHIA) CD45 2LMO : RL1LOg - NDC

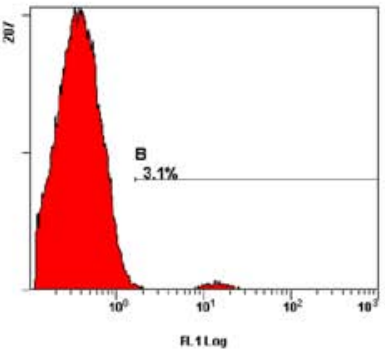

CD45

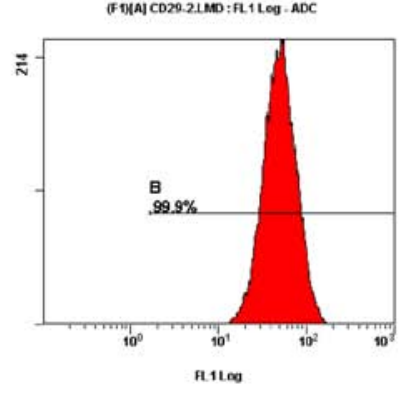

CD29

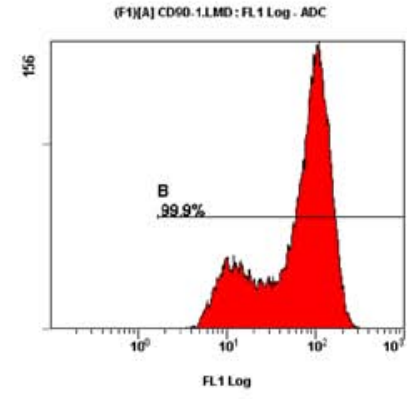

CD90

Figure 1. The culture and identification of BMSCs. (A) The morphology of BMSCs (magnification, x200). Scale bar, $100 \mu \mathrm{m}$. (B) The identification of BMSCs by flow cytometry with CD19, CD45, CD29, and CD90 antibodies. BMSCs, bone marrow-derived mesenchymal stem cells.

A
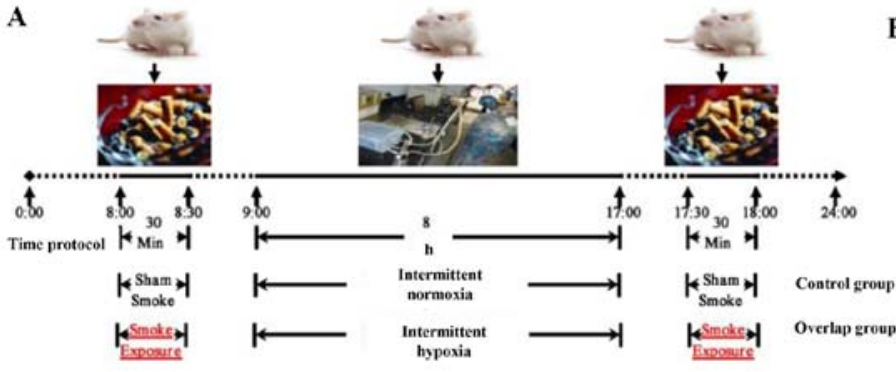

C

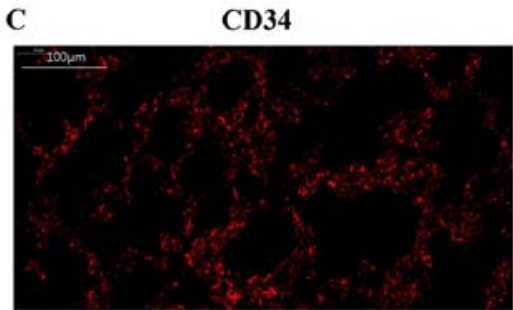

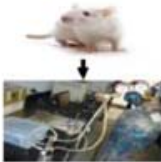

GFP labled-BMSCs

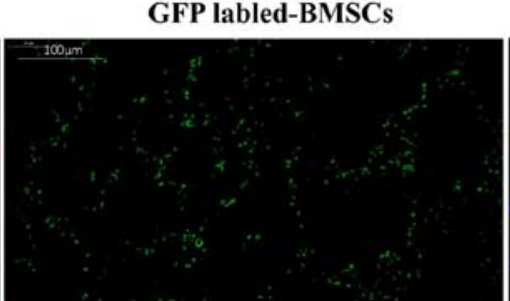

B
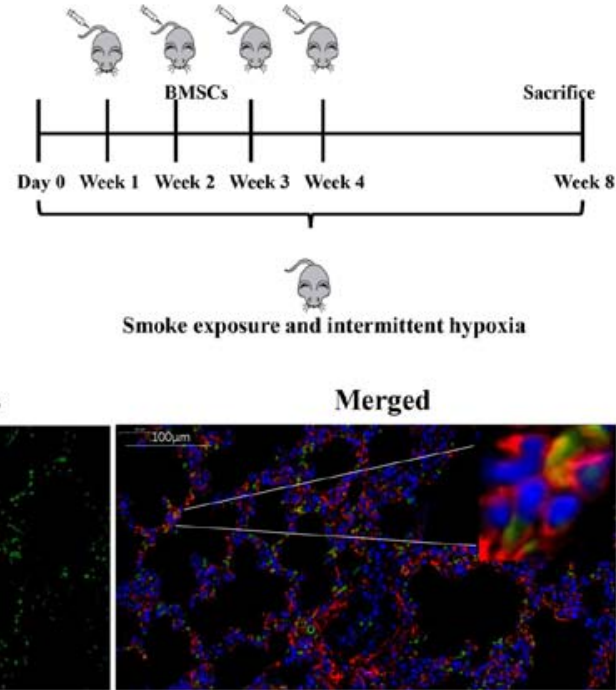

Figure 2. The location of BMSCs in lung tissues of OS rats. (A) The OS model profile for each day. (B) Experimental scheme of the BMSC transplantation. (C) BMSCs (green) and CD34 (red) expression in lung tissue under a fluorescence microscope (magnification, x400). Scale bar, $100 \mu \mathrm{m}$. Nuclei are stained with DAPI. BMSCs, bone marrow-derived mesenchymal stem cells; OS, overlap syndrome.

and intermittent cough. After 2 3 weeks, rats showed fur with yellowish color which easily fell off, a dispirited demeanor, a decline in appetite, an emaciated bodily form, and a decrease in activity ability. To evaluate the effect of BMSCs, we used an OS model with intravenous injection of BMSCs (Fig. 2B). GFP-labeled BMSCs showed green fluorescence, and green fluorescent cells (BMSCs) were observed in the alveolar walls of lung tissues in the OS+BMSC group (Fig. 2C). Immunofluorescence detection found that some cells had both green and red fluorescent (CD34-positive) cells (Fig. 2C).
There are three important cell types in alveolar tissues: Alveolar type I epithelial cells, alveolar type II epithelial cells and endotheliocytes. These three types of cells are important for maintaining the integrity of alveolar tissue, and the loss of one of these cell types directly affects the integrity of the alveolar tissue. CD34 is a marker of endotheliocytes; thus, we believe that some of the transplanted BMSCs had differentiated into endotheliocytes of the alveolar walls. Our results suggest that BMSCs can migrate into alveolar walls and differentiate into the endotheliocytes of alveolar walls. 
Table I. SOD activity and MDA content in the different rat groups in the OS model (mean \pm SD).

\begin{tabular}{lcccc}
\hline & \multicolumn{2}{c}{ Serum } & \multicolumn{2}{c}{ Lung tissues } \\
\cline { 2 - 3 } Rat groups & SOD $(\mathrm{U} / \mathrm{ml})$ & MDA $(\mathrm{nmol} / \mathrm{ml})$ & & SOD $(\mathrm{U} / \mathrm{ml})$ \\
\hline Control & $102.13 \pm 5.12$ & $6.07 \pm 2.12$ & $139.64 \pm 9.12$ & $5.73 \pm 63$ \\
OS & $26.43 \pm 1.33^{\mathrm{a}}$ & $12.32 \pm 1.72^{\mathrm{a}}$ & $43.12 \pm 1.87^{\mathrm{a}}$ & $10.43 \pm 2.11^{\mathrm{a}}$ \\
OS+BMSC & $93.84 \pm 2.08^{\mathrm{b}}$ & $7.23 \pm 0.75^{\mathrm{b}}$ & $129.32 \pm 1.87^{\mathrm{b}}$ & $5.98 \pm 1.2^{\mathrm{b}}$ \\
\hline
\end{tabular}

${ }^{\mathrm{a}} \mathrm{P}<0.001$, vs. the control group; ${ }^{\mathrm{b}} \mathrm{P}<0.001$, vs. the OS group. OS, overlap syndrome; BMSCs, bone marrow-derived mesenchymal stem cells; SOD, superoxide dismutase; MDA, malondialdehyde.

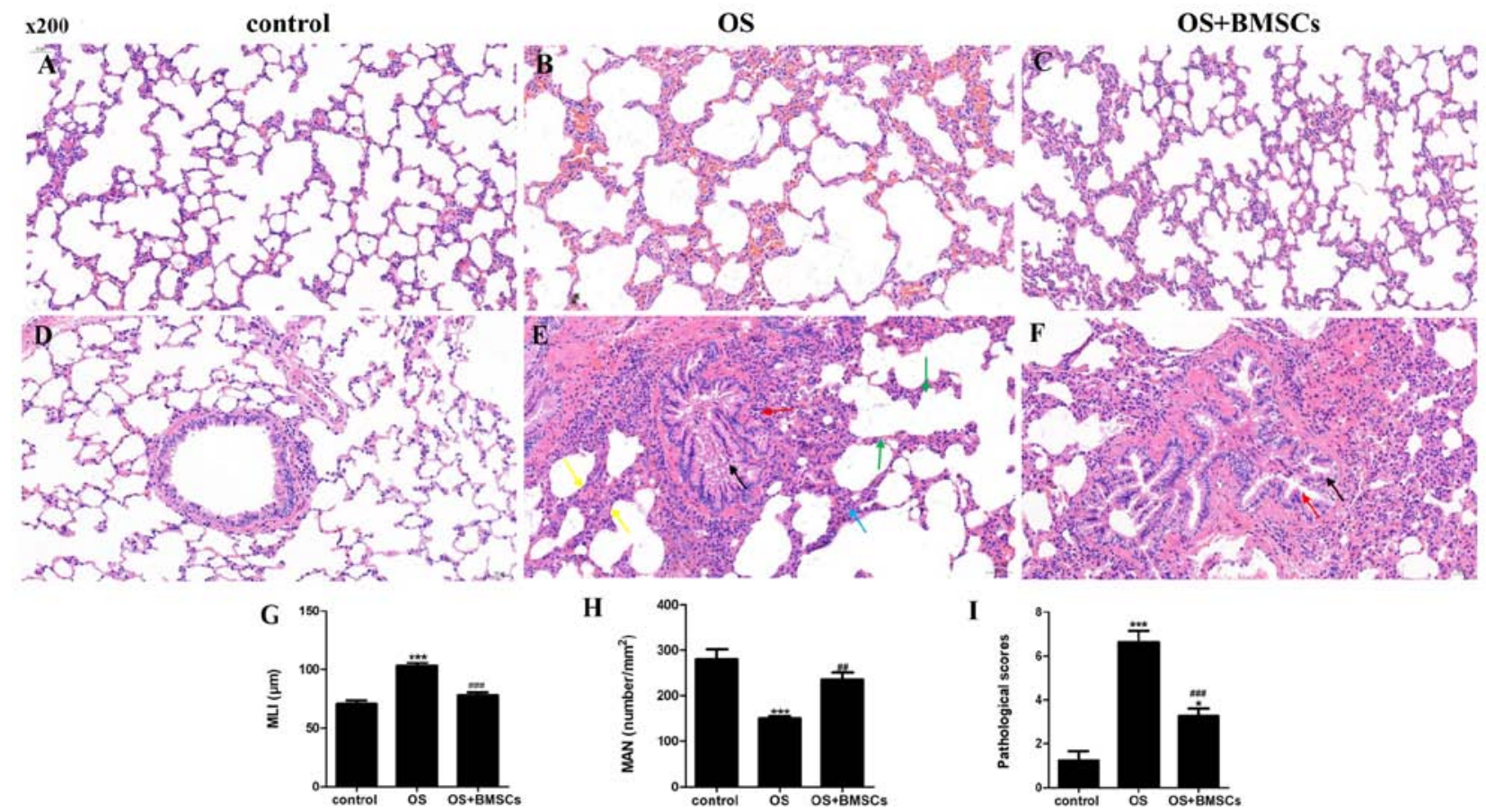

Figure 3. The therapeutic effects of BMSCs on OS model rats. Hematoxylin and eosin (H\&E) staining of lung tissue sections in the control group (A and D), OS group (B and E) and OS+BMSC group (C and F). Scale bar, $50 \mu \mathrm{m}$. (A) Alveolar structure was intact. (B) Alveolar size was different, the alveolar number was decreased, the alveolar space was enlarged and the alveolar septum was fractured. (C) Less enlarged alveolar space and less alveolar walls were thickened. (D) Alveolar structure was intact, and no inflammatory cell infiltration was observed. (E) A small number of bronchial epithelial cells were shed, and the bronchial epithelial cells exhibited edema and degeneration; the bronchial tubes were irregular in shape and narrow in the lumen; alveolar walls were thickened; the alveoli fused into a large cystic cavity; and much inflammatory cell infiltration. (F) Decreased inflammatory cell infiltration. Histological assessment of the sections was determined using the MLI (G), MAN (H) and pathological scores (I). A small number of bronchial epithelial cells are shed in the field, as shown by the black arrow. More bronchial epithelial cells showed edema and degeneration, cell swelling, loose cytoplasm and light staining, as shown by red arrows. Thickening of the alveolar walls is noted in the field, as shown by yellow arrows. The alveoli fused into a large cystic cavity as shown by green arrows. Inflammatory cells infiltrate the alveolar walls as shown by a blue arrow. ${ }^{* * * *} \mathrm{P}<0.001$ vs. control group; ${ }^{\# \#} \mathrm{P}<0.01$ and ${ }^{\# \# \# ~} \mathrm{P}<0.001$ vs. OS group. BMSCs, bone marrow-derived mesenchymal stem cells; OS, overlap syndrome; MLI, mean linear intercept; MAN, mean alveolar number.

BMSC transplantation reduces emphysematous changes in the OS rat model. H\&E staining was performed to detect emphysema and pathological scores in the lung tissues of rats. We quantified the enlargement of the alveolar spaces and alveolar septum fracture by MLI, MAN and the pathological scores in the lung tissue by the lung injury score. The alveolar structure was intact in the control group (Fig. 3A and D). In the OS group, there were emphysematous changes, the alveolar size was different, the alveolar number was decreased, the alveolar space was enlarged, and the alveolar septum was fractured (Fig. 3B). Moreover, in the OS group, a small number of bronchial epithelial cells were shed, and the bronchial epithelial cells exhibited edema and degeneration; the bronchial tubes were irregular in shape and narrow in the lumen; the alveolar walls were thickened; the alveoli fused into a large cystic cavity; and inflammatory cell infiltration in bronchial and lung tissue was observed in the OS group (Fig. 3E). In the OS+BMSC group, the inflammatory cell infiltration and emphysematous changes were significantly reduced after BMSC transplantation compared with before BMSC transplantation (Fig. 3C and F).

The MLI and pathological scores were significantly increased, and the MAN was significantly decreased in the OS group compared with the control group. Compared with the OS group, the MLI and pathological scores were significantly reduced and 

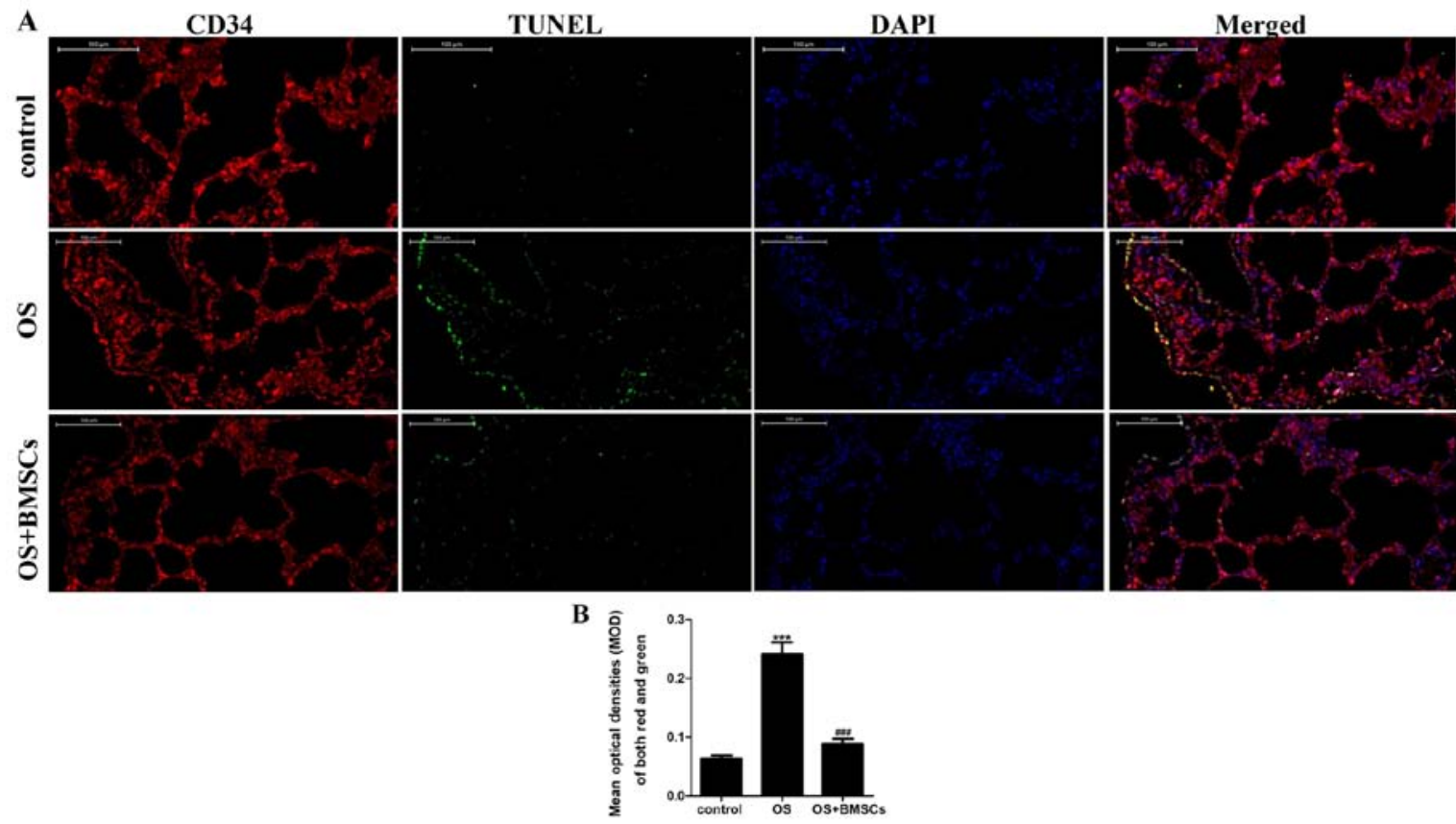

Figure 4. The effects of BMSCs on the endotheliocytes of alveolar walls in the OS+BMSC group rats. (A) The apoptosis of endotheliocytes in alveolar walls was detected by TUNEL assay. Green fluorescence shows apoptotic cells by a TUNEL assay. Red fluorescence shows the endotheliocytes by a CD34 immunofluorescence assay (magnification, $\mathrm{x} 400$ ). Scale bar, $100 \mu \mathrm{m}$. Nuclei are stained with DAPI. (B) Quantitative analysis of the co-expression of green and red fluorescence, indicating the apoptotic endotheliocytes in the alveolar walls. ${ }^{* * * *} \mathrm{P}<0.001$, vs. control group; ${ }^{\sharp \# \#} \mathrm{P}<0.001$, vs. OS group.

the MAN was promoted in the OS+BMSC group (Fig. 3G-I). BMSC transplantation alleviated lung injury in the OS rats.

BMSC transplantation has an antioxidant effect on OS rats. We also investigated the functions of BMSCs on oxidative stress in OS rats. As shown in Table I, there was an increase in the MDA content and a decrease in SOD activity in serum and lung tissues in the OS group, while BMSCs attenuated the elevated MDA content and activation of SOD.

BMSC transplantation reduces endotheliocyte apoptosis in the alveolar walls of $O S$ rats. The apoptotic cells under fluorescence microscopy displayed green fluorescence, while the endotheliocytes under fluorescence microscopy fluoresced red. The co-expression of red and green fluorescence indicated apoptotic endotheliocytes. As shown in Fig. 4A, few apoptotic cells were observed in the alveolar wall of the control group, and apoptosis was more obvious in the OS group than it was in the control group. BMSC transplantation significantly reduced endotheliocyte apoptosis in the alveolar walls of OS rats. Quantitative analysis showed that the apoptosis index of endotheliocytes in the OS+BMSC group was significantly lower than that in the OS group (Fig. 4B). These results suggest that BMSCs can reduce endotheliocyte apoptosis in the alveolar walls of OS rats.

BMSC transplantation promotes the expression of CD31 and $V W F$ in the alveolar walls of OS rats. CD31 and VWF are markers of endotheliocytes. The levels of CD31 and VWF were detected by an immunohistochemical assay. As shown in Fig. 5A and C, the cytoplasm of alveolar wall cells in the lung tissue sections of the transplanted rats was stained brown. A small amount of brown was observed in the alveolar wall of the OS group compared with the control group. The quantitative results (Fig. 5B and D) of the immunohistochemical assay are consistent with Fig. 5A and C. BMSC transplantation significantly increased the brown staining in the alveolar walls of OS rats. These results indicate that BMSCs suppressed the apoptosis of endotheliocytes in the alveolar walls of OS rats.

\section{Discussion}

Several studies have confirmed that individuals with chronic obstructive pulmonary disease (COPD) and obstructive sleep apnea (OSA) have more profound nocturnal oxygen desaturation and sleep disturbances compared with those with either disease alone (31). Marin et al (5) found decreased survival among patients with overlap syndrome (OS) compared to those with either COPD or OSA alone. Bone marrow-derived mesenchymal stem cells (BMSCs) are stem cells that are found in connective tissues throughout the whole body; they are easy to harvest, easy to culture, quick to proliferate and weakly immunogenic (32-34). Previous studies have shown that BMSCs can migrate into damaged lung tissue $(35,36)$. Moreover, BMSCs not only differentiate into lung epithelial cells in damaged lung tissue (37) but also secrete various growth factors and cytokines in response to lung injury (38). Some studies have focused on the effect of BMSCs on COPD alone or OSA alone, but few studies have focused on the OS model.

One of the characteristics of OSA is intermittent hypoxemia, and smoking is the main cause of COPD development. Thus, we constructed the OS rat model with exposure to cigarette smoke and intermittent hypoxia, yet the intermittent hypoxia model cannot simulate upper airway obstruction. At present, there are no relevant studies on spontaneous and 

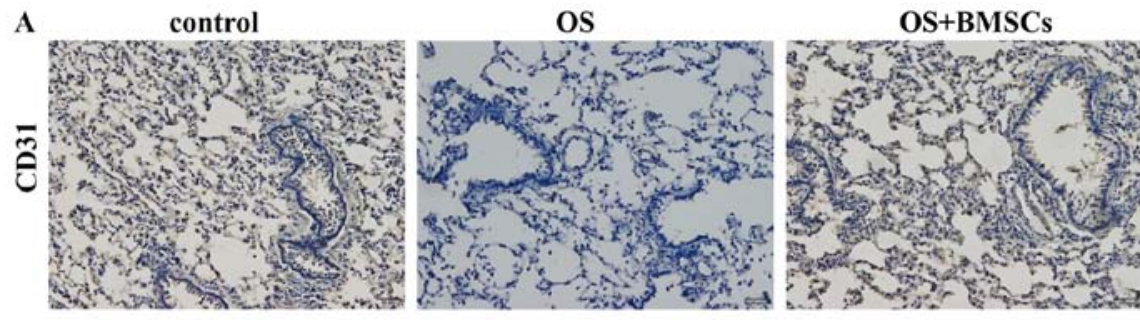

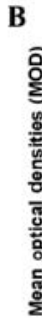
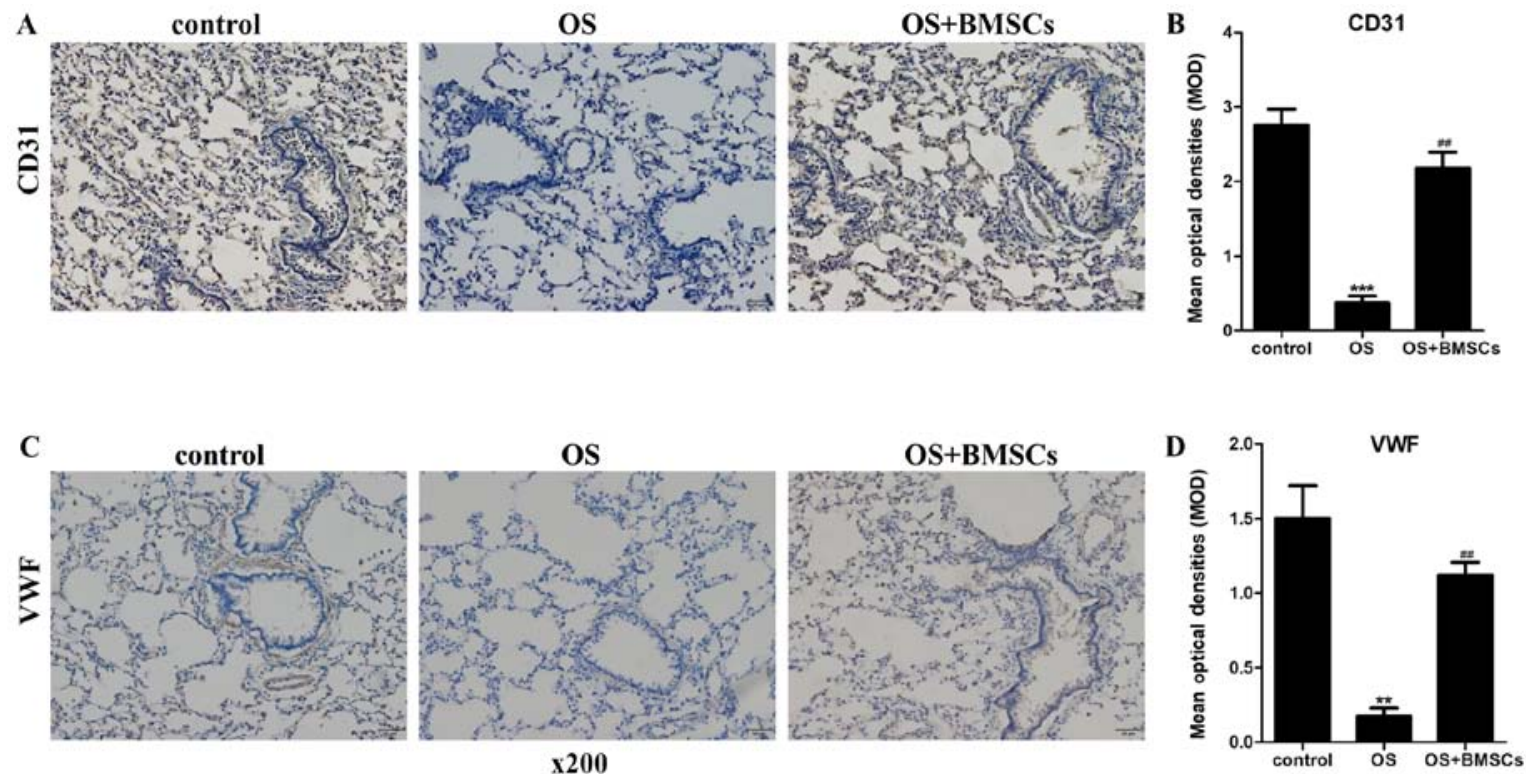

Figure 5. The effects of BMSCs on CD31 and VWF levels in alveolar walls in the OS+BMSC group rats. (A and C) The expression levels of CD31 and VWF were detected by an immunohistochemical assay (magnification, $\mathrm{x} 400$ ). Scale bar, $50 \mu \mathrm{m}$. (A) Brown staining of cells in the control group. A small amount of brown was observed in the alveolar wall of the OS group, and increasing brown staining of cells in the OS+BMSCs group. (B) Quantitative results of the CD31 protein immunohistochemical assay. (C) A large number of stained cells was observed in the control group, less brown was observed in the alveolar wall of the OS group, and increased brown staining of cells was observed in the OS+BMSCs group. (D) Quantitative results of the VWF protein immunohistochemical assay. The brown color shows the positive cells for $\mathrm{CD} 31$ and VWF. The blue color shows the nuclei. ${ }^{* * *} \mathrm{P}<0.01$ and ${ }^{* * * *} \mathrm{P}<0.001$ vs. control group; ${ }^{\sharp \#} \mathrm{P}<0.001$, vs. OS group.

natural OS animal models, and there is no accepted evaluation standard for the OS model. Theoretically, the establishment of the model can be judged based on the evaluation criteria of OSA and COPD animal models, and the main pathological characteristics of the two can determine the success of the model. Building an ideal OS animal model requires more exploration and practice. Compared with the control group, the OS rats were in poor condition, with acidosis, hypoxemia and carbon dioxide retention, and right ventricular hypertrophy (data not shown). In this study, we first examined the effects of BMSC transplantation in the OS model induced by cigarette smoke and intermittent hypoxia and its potential mechanisms in vitro. Our results revealed that BMSC transplantation relieved emphysema and lung injury in the OS model by inhibiting oxidative stress and apoptosis of endotheliocytes in the alveolar walls. We created an OS model induced by cigarette smoke and intermittent hypoxia. Alveolar wall rupture and emphysematous changes were observed in the OS group. The MLI and pathological scores were higher and the MAN was lower in the OS group than these parameters in the control group. Moreover, the malondialdehyde (MDA) content was increased and the superoxide dismutase (SOD) activity was decreased in the OS group compared with the control group. These results suggest that cigarette smoke and intermittent hypoxia could promote oxidative stress, emphysema, alveolar congestion, alveolar wall edema and inflammatory cell infiltration and increase the lung injury score. The BMSC transplantation group exhibited significantly alleviated lung injury compared with the OS group. SOD is an important free radical scavenger in the body that can protect cell membranes from oxidation and lipid peroxidation (39). Decreased SOD activity in blood and lung tissues is one of the manifestations of smoking-induced COPD damage. MDA is a product of lipid peroxidation induced by free radical attack on biofilms. The MDA level can reflect the degree of lipid peroxidation in the body (40), and its level in serum and lung can indirectly reflect the degree of cell damage. The increased SOD activity and decreased MDA content in the serum and lung of the OS+BMSC group rats, suggest that BMSCs can enhance the activity of free radical scavenging enzymes and enhance the antioxidant capacity and reduce the damage caused by lipid peroxidation.

To investigate whether the transplanted BMSCs could migrate into the lung in OS rats, we marked BMSCs with an eGFP lentivirus before injection. We also detected endotheliocytes by CD34 immunofluorescence. We observed green fluorescence in the alveolar walls and its co-expression with CD34. This implied that BMSCs can migrate to the injury lung tissues, but not only lung tissues. We found that BMSCs also can migrate into the aorta (data not shown). Moreover, decreased apoptosis of endotheliocytes in alveolar walls was observed in the OS+BMSC group, and a high rate of apoptosis was found in the OS group. These results suggest that BMSCs can migrate into lung tissues and differentiate into endotheliocytes in alveolar walls, and BMSC transplantation markedly reduced the apoptosis of endotheliocytes in alveolar walls, which may represent one mechanism for emphysema therapy. Some studies have indicated that the apoptosis of alveolar type II epithelial cells is directly associated with emphysema $(41,42)$ and that MSCs can differentiate into alveolar type I and II epithelial cells (43-45). These studies indicate that MSCs can differentiate into alveolar cells. In the present study, transplanted BMSCs with CD34-positive expression differentiated into alveolar wall endotheliocytes, and the apoptosis of endotheliocytes in the alveolar walls is important for OS, which may help to alleviate emphysema. We also examined 
the expression of the endothelial cell markers CD31 and VWF by immunohistochemical assay. The results suggested that BMSCs suppress the apoptosis of endothelial cells, which agrees with previous TUNEL results. There was a possibility that the release of growth factors and structural support may be a determinant for the regenerative effects observed following treatment with BMSCs. Since BMSCs have strong proliferative properties, they are theoretically tumorigenic in tissue or cell transplantation (46). Therefore, the numbers of BMSCs for treatment should be strictly controlled, so that the excessive accumulation of BMSCs do not cause negative effects. BMSCs can migrate to damaged tissues, so they are present not only in the lungs, but also in other tissues, such as the aorta and the heart. This indicates that the OS model can lead not only to lung injury but also to other organ injury. We will study the repair effect of BMSCs on other organs in OS rats in further research. There is also the question of whether the presence of undifferentiated BMSCs causes damage to organs, since excessive injection increases the risk of tumors. Therefore, the use of BMSCs must be strictly controlled within a safe range, which requires more in-depth research.

In summary, we demonstrated the therapeutic effect of BMSC administration in OS rats induced by cigarette smoke and intermittent hypoxia. BMSCs can suppress pulmonary emphysema and oxidative stress in OS rats. Moreover, BMSCs were able to migrate into the alveolar walls and differentiate into endotheliocytes in the alveolar wall, inhibiting endotheliocyte apoptosis. This experiment confirms that the inhibition of the progression of emphysema by BMSCs in the OS model may be through the differentiation of BMSCs into endotheliocytes consequently suppressing endotheliocyte apoptosis and by their antioxidative stress function. However, further elucidation of the relationship between BMSCs and endotheliocyte proliferation and whether they can promote endotheliocyte proliferation remain to be further studied. Our study indicates that BMSCs are potent and may serve as a basis for clinical trials with OS patients in the near future.

\section{Acknowledgements}

Not applicable.

\section{Funding}

This research was supported by the National Natural Science Foundation of China (no. 81560010) and the Science and Technology Planning Project of Yunnan Province [no. 2017FE467(-100)].

\section{Availability of data and materials}

The datasets used and/or analyzed during the current study are available from the corresponding author on reasonable request.

\section{Authors' contributions}

$\mathrm{MC}, \mathrm{ZH}$ and $\mathrm{ZJ}$ conceived and designed the study. HB, XP, JH, $\mathrm{LH}$ and $\mathrm{XH}$ performed the experiments. JD, KZ, LW, QW and $\mathrm{XG}$ collected and processed the data. $\mathrm{MC}, \mathrm{ZH}$ and $\mathrm{QW}$ wrote the manuscript. $\mathrm{MC}, \mathrm{ZH}, \mathrm{XG}$ and $\mathrm{ZJ}$ reviewed and edited the manuscript. All authors read and approved the manuscript and agree to be accountable for all aspects of the research in ensuring that the accuracy or integrity of any part of the work are appropriately investigated and resolved.

\section{Ethics approval and consent to participate}

The animal experiments were approved by the Animals Ethics Committee of Kunming Medical University and the Research Medical Ethics Committee of Kunming Medical University.

\section{Patient consent for publication}

Not applicable.

\section{Competing interests}

The authors declare that they have no competing interests.

\section{References}

1. McNicholas WT, Verbraecken J and Marin JM: Sleep disorders in COPD: The forgotten dimension. Eur Respir Rev 22: 365-375, 2013.

2. Rabe KF, Hurd S, Anzueto A, Barnes PJ, Buist SA, Calverley P, Fukuchi Y, Jenkins C, Rodriguez-Roisin R, van Weel C, et al: Global strategy for the diagnosis, management, and prevention of chronic obstructive pulmonary disease: GOLD executive summary. Am J Respir Crit Care Med 176: 532-555, 2007.

3. Alkhalil M, Schulman E and Getsy J: Obstructive sleep apnea syndrome and asthma: What are the links? J Clin Sleep Med 5: 71-78, 2009.

4. Flenley DC: Sleep in chronic obstructive lung disease. Clin Chest Med 6: 651-661, 1985.

5. Marin JM, Soriano JB, Carrizo SJ, Boldova A and Celli BR: Outcomes in patients with chronic obstructive pulmonary disease and obstructive sleep apnea: The overlap syndrome. Am J Respir Crit Care Med 182: 325-331, 2010.

6. Neilan TG, Bakker JP, Sharma B, Owens RL, Farhad H, Shah RV, Abbasi SA, Kohli P, Wilson J, DeMaria A, et al: T1 measurements for detection of expansion of the myocardial extracellular volume in chronic obstructive pulmonary disease. Can J Cardiol 30: 1668-1675, 2014

7. Sharma B, Neilan TG, Kwong RY, Mandry D, Owens RL, McSharry D, Bakker JP and Malhotra A: Evaluation of right ventricular remodeling using cardiac magnetic resonance imaging in co-existent chronic obstructive pulmonary disease and obstructive sleep apnea. COPD 10: 4-10, 2013.

8. Pittenger MF, Mackay AM, Beck SC, Jaiswal RK, Douglas R, Mosca JD, Moorman MA, Simonetti DW, Craig S and Marshak DR: Multilineage potential of adult human mesenchymal stem cells. Science 284: 143-147, 1999.

9. Antoniou KM,Karagiannis K, Tsitoura E, Bibaki E,LasithiotakiI, Proklou A, Spandidos DA and Tzanakis N: Clinical applications of mesenchymal stem cells in chronic lung diseases. Biomed Rep 8: 314-318, 2018.

10. Gupta N, Su X, Popov B, Lee JW, Serikov V and Matthay MA: Intrapulmonary delivery of bone marrow-derived mesenchymal stem cells improves survival and attenuates endotoxin-induced acute lung injury in mice. J Immunol 179: 1855-1863, 2007.

11. Ishizawa K, Kubo H, Yamada M, Kobayashi S, Numasaki M, Ueda S, Suzuki T and Sasaki H: Bone marrow-derived cells contribute to lung regeneration after elastase-induced pulmonary emphysema. FEBS Lett 556: 249-252, 2004.

12. Spees JL, Pociask DA, Sullivan DE, Whitney MJ, Lasky JA, Prockop DJ and Brody AR: Engraftment of bone marrow progenitor cells in a rat model of asbestos-induced pulmonary fibrosis. Am J Respir Crit Care Med 176: 385-394, 2007.

13. Caples SM, Garcia-Touchard A and Somers VK: Sleep-disordered breathing and cardiovascular risk. Sleep 30: 291-303, 2007.

14. Campana L, Eckert DJ, Patel SR and Malhotra A: Pathophysiology \& genetics of obstructive sleep apnoea. Indian J Med Res 131: 176-187, 2010. 
15. Jelic S, Padeletti M, Kawut SM, Higgins C, Canfield SM, Onat D, Colombo PC, Basner RC, Factor P and LeJemtel TH: Inflammation, oxidative stress, and repair capacity of the vascular endothelium in obstructive sleep apnea. Circulation 117: 2270-2278, 2008.

16. Carreras A, Almendros I and Farré R: Potential role of bone marrow mesenchymal stem cells in obstructive sleep apnea. Int J Stem Cells 4: 43-49, 2011.

17. Park JS, Kim HK, Kang EY, Cho R and Oh YM: Potential therapeutic strategy in chronic obstructive pulmonary disease using pioglitazone-augmented wharton's jelly-derived mesenchymal stem cells. Tuberc Respir Dis (Seoul) 82: 158-165, 2019.

18. Liu HM, Liu YT, Zhang J and Ma LJ: Bone marrow mesenchymal stem cells ameliorate lung injury through anti-inflammatory and antibacterial effect in COPD mice. J Huazhong Univ Sci Technolog Med Sci 37: 496-504, 2017.

19. Miglino N, Roth $M$, Tamm M and Borger P: Asthma and COPD-the C/EBP connection. Open Respir Med J 6: 1-13, 2012.

20. Pryor WA and Stone K: Oxidants in cigarette smoke. Radicals, hydrogen peroxide, peroxynitrate, and peroxynitrite. Ann N Y Acad Sci 686: 12-28, 1993.

21. Antus B, Harnasi G, Drozdovszky O and Barta I: Monitoring oxidative stress during chronic obstructive pulmonary disease exacerbations using malondialdehyde. Respirology 19: 74-79, 2014.

22. Cristóvão C, Cristóvão L, Nogueira F and Bicho M: Evaluation of the oxidant and antioxidant balance in the pathogenesis of chronic obstructive pulmonary disease. Rev Port Pneumol 19: 70-75, 2013 (In English, Portuguese).

23. Montaño M, Cisneros J, Ramírez-Venegas A, Pedraza-Chaverri J, Mercado D, Ramos C and Sansores RH: Malondialdehyde and superoxide dismutase correlate with FEV(1) in patients with COPD associated with wood smoke exposure and tobacco smoking. Inhal Toxicol 22: 868-874, 2010.

24. Hodge S, Hodge G, Holmes M and Reynolds PN: Increased airway epithelial and T-cell apoptosis in COPD remains despite smoking cessation. Eur Respir J 25: 447-454, 2005.

25. Zhen G, Xue Z, Zhao J, Gu N, Tang Z, Xu Y and Zhang Z: Mesenchymal stem cell transplantation increases expression of vascular endothelial growth factor in papain-induced emphysematous lungs and inhibits apoptosis of lung cells. Cytotherapy 12: 605-614, 2010

26. Zhao Y, Xu A, Xu Q, Zhao W, Li D, Fang X and Ren Y: Bone marrow mesenchymal stem cell transplantation for treatment of emphysemic rats. Int J Clin Exp Med 7: 968-972, 2014.

27. Lee JH, Lee DS, Kim EK, Choe KH, Oh YM, Shim TS, Kim SE, Lee YS and Lee SD: Simvastatin inhibits cigarette smoking-induced emphysema and pulmonary hypertension in rat lungs. Am J Respir Crit Care Med 172: 987-993, 2005.

28. Thurlbeck WM: Measurement of pulmonary emphysema. Am Rev Respir Dis 95: 752-764, 1967.

29. Zhen G, Liu H, Gu N, Zhang H, Xu Y and Zhang Z: Mesenchymal stem cells transplantation protects against rat pulmonary emphysema. Front Biosci 13: 3415-3422, 2008.

30. Vernooy JH, Dentener MA, van Suylen RJ, Buurman WA and Wouters EF: Long-term intratracheal lipopolysaccharide exposure in mice results in chronic lung inflammation and persistent pathology. Am J Respir Cell Mol Biol 26: 152-159, 2002.

31. Sanders MH, Newman AB, Haggerty CL, Redline S, Lebowitz M, Samet J, O'Connor GT, Punjabi NM and Shahar E; Sleep Heart Health Study: Sleep and sleep-disordered breathing in adults with predominantly mild obstructive airway disease. Am J Respir Crit Care Med 167: 7-14, 2003.

32. Kashiwakura Y, Katoh Y, Tamayose K, Konishi H, Takaya N, Yuhara S, Yamada M, Sugimoto K and Daida H: Isolation of bone marrow stromal cell-derived smooth muscle cells by a human SM22alpha promoter: In vitro differentiation of putative smooth muscle progenitor cells of bone marrow. Circulation 107: 2078-2081, 2003.
33. Devine SM, Cobbs C, Jennings M, Bartholomew A and Hoffman R: Mesenchymal stem cells distribute to a wide range of tissues following systemic infusion into nonhuman primates. Blood 101: 2999-3001, 2003.

34. Bos C, Delmas Y, Desmoulière A, Solanilla A, Hauger O, Grosset C, Dubus I, Ivanovic Z, Rosenbaum J, Charbord P, et al: In vivo MR imaging of intravascularly injected magnetically labeled mesenchymal stem cells in rat kidney and liver. Radiology 233: 781-789, 2004.

35. Yamada M, Kubo H, Kobayashi S, Ishizawa K, Numasaki M, Ueda S, Suzuki T and Sasaki H: Bone marrow-derived progenitor cells are important for lung repair after lipopolysaccharide-induced lung injury. J Immunol 172: 1266-1272, 2004.

36. Ortiz LA, Gambelli F, McBride C, Gaupp D, Baddoo M, Kaminski N and Phinney DG: Mesenchymal stem cell engraftment in lung is enhanced in response to bleomycin exposure and ameliorates its fibrotic effects. Proc Natl Acad Sci USA 100: 8407-8411, 2003.

37. Kotton DN, Ma BY, Cardoso WV, Sanderson EA, Summer RS, Williams MC and Fine A: Bone marrow-derived cells as progenitors of lung alveolar epithelium. Development 128: 5181-5188, 2001.

38. Weiss DJ, Kolls JK, Ortiz LA, Panoskaltsis-Mortari A and Prockop DJ: Stem cells and cell therapies in lung biology and lung diseases. Proc Am Thorac Soc 5: 637-667, 2008.

39. Chandra Jagetia G, Rajanikant GK, Rao SK and Shrinath Baliga M: Alteration in the glutathione, glutathione peroxidase, superoxide dismutase and lipid peroxidation by ascorbic acid in the skin of mice exposed to fractionated gamma radiation. Clin Chim Acta 332: 111-121, 2003.

40. Zhang RQ, Li DY, Xu TD, Zhu SS, Pan HJ, Fang F, Wu X and Sun H: Antioxidative effect of luteolin pretreatment on simulated ischemia/reperfusion injury in cardiomyocyte and perfused rat heart. Chin J Integr Med 23: 518-527, 2017.

41. Mauck RL, Yuan X and Tuan RS: Chondrogenic differentiation and functional maturation of bovine mesenchymal stem cells in long-term agarose culture. Osteoarthritis Cartilage 14: 179-189, 2006.

42. Snykers S, Vanhaecke T, Papeleu P, Luttun A, Jiang Y, Vander Heyden Y, Verfaillie C and Rogiers V: Sequential exposure to cytokines reflecting embryogenesis: The key for in vitro differentiation of adult bone marrow stem cells into functional hepatocyte-like cells. Toxicol Sci 94: 330-341, discussion 235-239, 2006.

43. Grove JE, Lutzko C, Priller J, Henegariu O, Theise ND, Kohn DB and Krause DS: Marrow-derived cells as vehicles for delivery of gene therapy to pulmonary epithelium. Am J Respir Cell Mol Biol 27: 645-651,2002.

44. Abe S, Lauby G, Boyer C, Rennard SI and Sharp JG: Transplanted $\mathrm{BM}$ and $\mathrm{BM}$ side population cells contribute progeny to the lung and liver in irradiated mice. Cytotherapy 5: 523-533, 2003.

45. Rojas M, Xu J, Woods CR, Mora AL, Spears W, Roman J and Brigham KL: Bone marrow-derived mesenchymal stem cells in repair of the injured lung. Am J Respir Cell Mol Biol 33: 145-152, 2005.

46. Liu CJ, Kuo FC, Hu HM, Chen CY, Huang YB, Cheng KH, Yokoyama KK, Wu DC, Hsieh S and Kuo CH: 17ß-Estradiol inhibition of IL-6-Src and Cas and paxillin pathway suppresses human mesenchymal stem cells-mediated gastric cancer cell motility. Transl Res 164: 232-243, 2014.

(i) $\Theta$ This work is licensed under a Creative Commons Attribution-NonCommercial-NoDerivatives 4.0 International (CC BY-NC-ND 4.0) License. 